K. Mitsui, Y. Muraki ${ }^{*}$, A. Okada, Y. Ohashi

Institute for Cosmic Ray Research, Univ. of Tokyo, Tanashi, Tokyo 188

S. Matsumoto, R. Tanaka

Dep. of Physics, Chuo Univ., Bunkyo, Tokyo 112

H. Iwasaki

National High Energy Lab., KEK, Tsukuba 305

I. Nakamura

Dep. Of Physics, Nagoya Univ., Nagoya 464

\title{
abstract
}

A new method for the energy determination of Tev muons will be studied at Tevatron beam line. More than 200 layers scintillation shower detector is located in front of Tevatron muon experimental group $(E-665)$. The energy resolution of small shower detector induced by muons will be experimentally obtained.

\section{Experimental purpose}

When a muon with $E_{\mu}$ higher than $\geqslant 1 \mathrm{TeV}$ passes through material, a small shower is inevitably associated with the muon. This causes a difficulty of the muon tracking, because many shower particles enter into the same wire chamber with the real muon track. \#)

Spokesman : Y. MURAKI 
It will become serious problem for the TeV muon detection, especially in the very forward region in an SSC experiment.

Here we propose to try anotiner method for the determination of muon energy. The technique was born in cosmic ray experiments involving the determination of the muon momentun wihtout using magnet. The technique is called "Pair meter".

The principle is as follows:

When the muon energy increases, direct electron pair production cross-section increases almost linearly with muon energy (Fig. 1). If we use this rising direct pair production cross-section, we have the possibility to determine the energy of the muons without using a magnet ${ }^{1 / 2)}$.

A few investigations have been made concerning this technique, either by Monte Carlo method ${ }^{3)}$ or experimentally ${ }^{4}$. However due to the limited number of high energy muons accepted by the cosmic ray detector, not enough experimental results have been obtained.

The technique is now not only necessary for the new underground non accelerator experiments but also for future accelerator experiments , i.e., for super-collider muon detection. The technique will open a new possibility of the muon detection at SSC, in case we use 1000 layers scintillation shower calorimeter (Fig. 2).

Therefore we propose here to investigate such possibility not only by Monte Carlo method, but also directly by actual experiment using the Tevatron muon beam ${ }^{5)}$. " since the Tevatron is the only machine in the world to produce $\mathrm{TeV}$ muons, it opens such a new possibility.

We also aim to measure the "minimum bias" small-q ${ }^{2}$ muon nuclear interaction cross-section by this experiment, changing the target material from iron to carbon. We can know the shadowing effect of the photo-nuclear interaction induced by muon by various nucleii. 
2. Experimental Method

We will use a kind of 'calorimeter technique' for this purpose, but an important difference is the number of the sampling points. The final number of sampling points has not yet been decided, because it depends on budget circumstances, but we can say at least we prepare $\$ 200$ layers ( and hopfully 500 layers ) sampling calorimeter. The detector will be located in a free space in the Tevatron muon zone, about 20 meters longitudinal distance (Fig. 3). We consider the thickness of the scintillator to be $1 \mathrm{~cm}$ and the size $20 \times 20 \mathrm{~cm}^{2}$. The size is enough to absorb the lateral distribution of electro-magnetic shower and also the nuclear shower (Fig. 4). It also matches the muon beam spread from Tevatron(Fig.5). We plan to construct three types of calorineter as listed below. The data taking is very standard. The light of scintillator is collected by fish-tail type light guides and sent to photomultipliers. Then the analogue signals are read by standard CAMAC ADC.

\begin{tabular}{ll}
\hline Type A) 200 scinti + lead plates & $\begin{array}{l}\text { total thickness of matter } \\
\text { B) } 200 \text { scinti + iron plates }\end{array}$ \\
& $8 \mathrm{~m}+2 \mathrm{~m}(2.5$ tons $)$ \\
C) 200 scinti + carbon plates & $10 \mathrm{~m}+2 \mathrm{~m}(1.8$ tons $)$
\end{tabular}
We draw the schematic view of the experiment in Fig. 6.

\section{Beam condition}

We request the following beam conditions (Table I ). The expected beam momentum spectrum is shown in Fig. 7. We request the measurement of scattered muon momentum and the scattered angle by $E-665$ group. 


\begin{tabular}{ccc}
$\begin{array}{c}\text { beam condition } \\
E_{\mathrm{u}}(\mathrm{GeV})\end{array}$ & $\begin{array}{c}\text { Table } \\
\text { \# of events }\end{array}$ & $\begin{array}{l}\text { Expected \# of nuclear interactiont } \\
200\end{array}$ \\
\hline & $20 \mathrm{~K}$ each & 100 each for $\mathrm{C}, \mathrm{Pb}, \mathrm{Fe}$ \\
400 & $200 \mathrm{~K}$ each & 1000 each for $\mathrm{C}, \mathrm{Pb}, \mathrm{Fe}$ \\
600 & $200 \mathrm{~K}$ eack & \\
800 & $200 \mathrm{~K}$ each
\end{tabular}

We estimate that we will need $\sim 20$ magnetic tapes $(6250 \mathrm{bpi})$. The number of events has been decided that we could get I $\mathrm{K}$ nuclear interaction in each series. For the sake of $\mu^{+} e^{-}$study, only $20 \mathrm{~K}$ events enough for each series, however for the descrimination of nuclear cascade from the showers, an investigation is necessary. $(\bar{I})$

\section{Data analysis}

The muon energy is obtained by counting the number of scintillators which "fire". For example if one scintillator records the number of electron greater than 30, we count "one' fired. The energy resolution for 500 layers obtained in such a way is presented in Fig. 8 (for $N_{e} \geq 10$ particles) and in Fig. 9 (for $N_{e} \geq 20$ particles in the scintillator).

We plan to improve our Monte Carlo by a three dimensional calculation. The real data will be compared to the three dimensional Monte Carlo results. Data analysis will be made in Japan and also in the USA.

\section{Japanese and US responsibility}

In Japan side, we will construct the calorimeter and ship it to Fermilab before Nov. 1986. Fermilab will have the responsibility for the data acquisition system and will also prepare the iron, lead and carbon plates. Fermilab will also measure the scattered muon momentum and angle. Both have the responsibility on the paper publication and in the shift time a few members from Japan will participate. 
Bibliography

1) Alexei and Zatsepin G.T. : Int. Conf on Cosmic Rays in Moscow 1 (1960), 324 .

2) F. Reins and S. Miyake : Discussions at Denver Int. Conf. on Cosmic Rays (1973).

3) T. Wadi and T. Kitamura : Propr. Theor. Physics, 41 (1969), 1587 M.K. Moe : Nouvo Cimento, 66B (1970), 90.

4) I. Nakamura et al.: Int. Conf. on Cosmic Rays in kyoto, $\underline{10}(1979), 19$.

5) Y. Muraki : Talk at Wisconsin for SSC muon detection (1985 Apr.) ICRR report- $125-85-5$ (1985) .

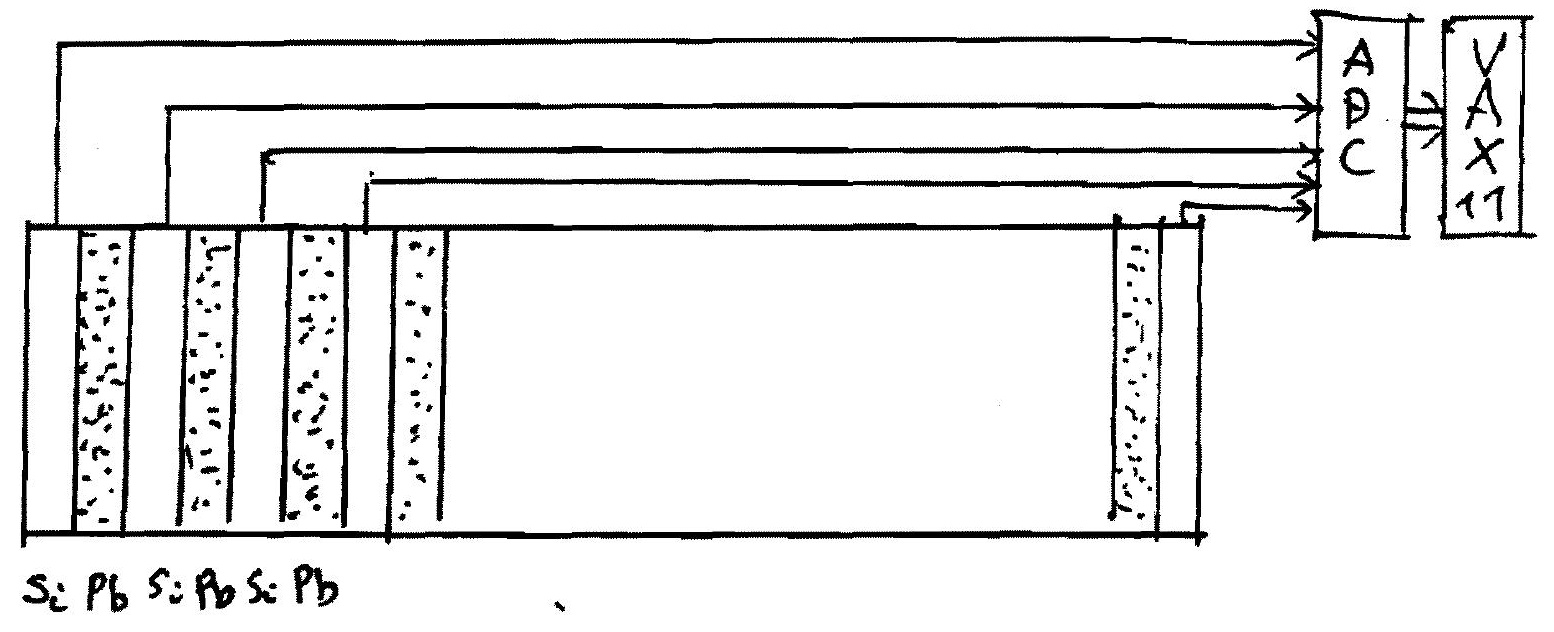

$$
\text { Fig. } 6
$$




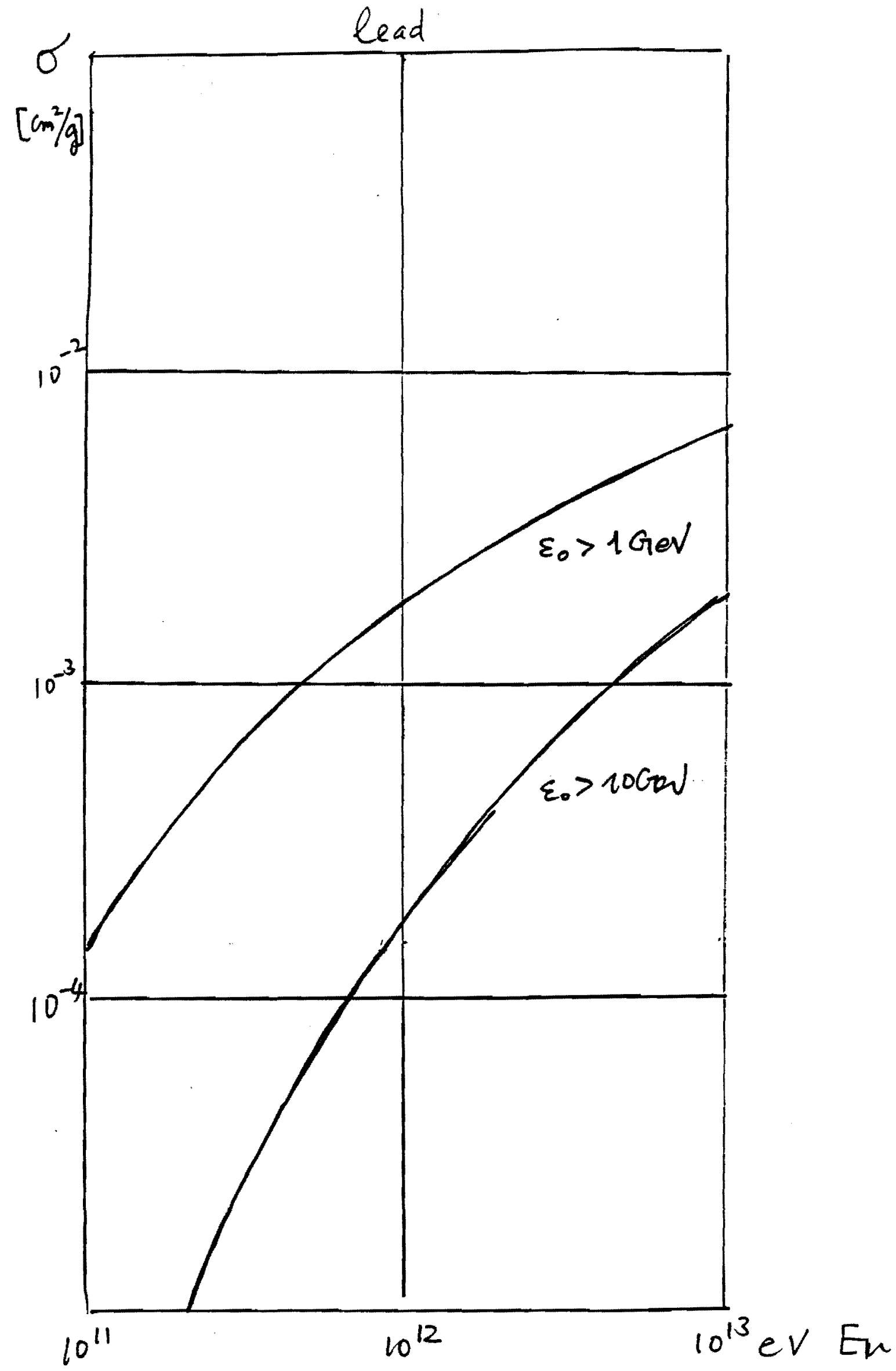




$$
=1 D M
$$


Fig. 3

3.
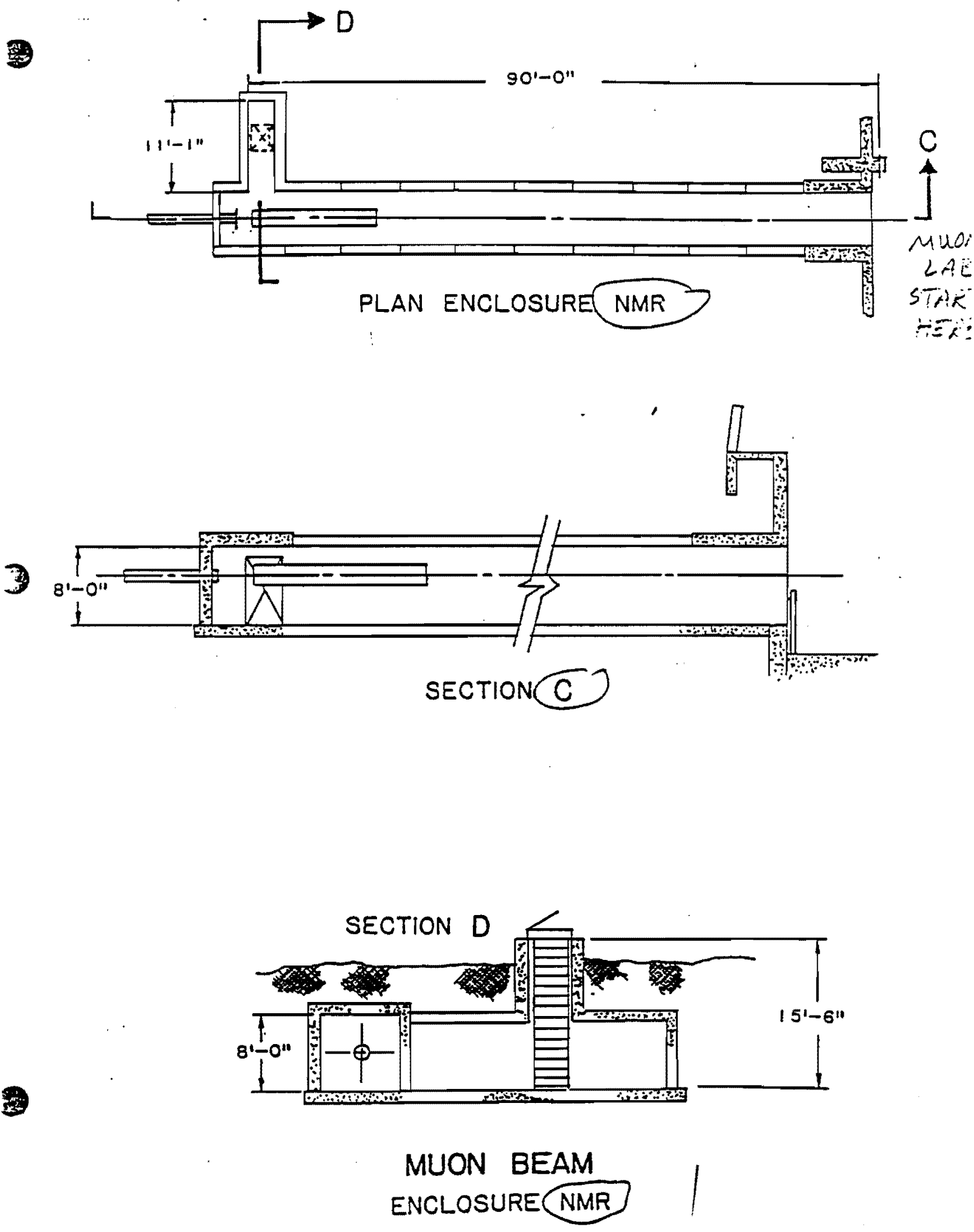


$$
\text { Fig. } 4
$$

trom:

Nuctear Instruments and Methods in Physics Research A236 (1985) 47-54

North-Holland, Ansterdam
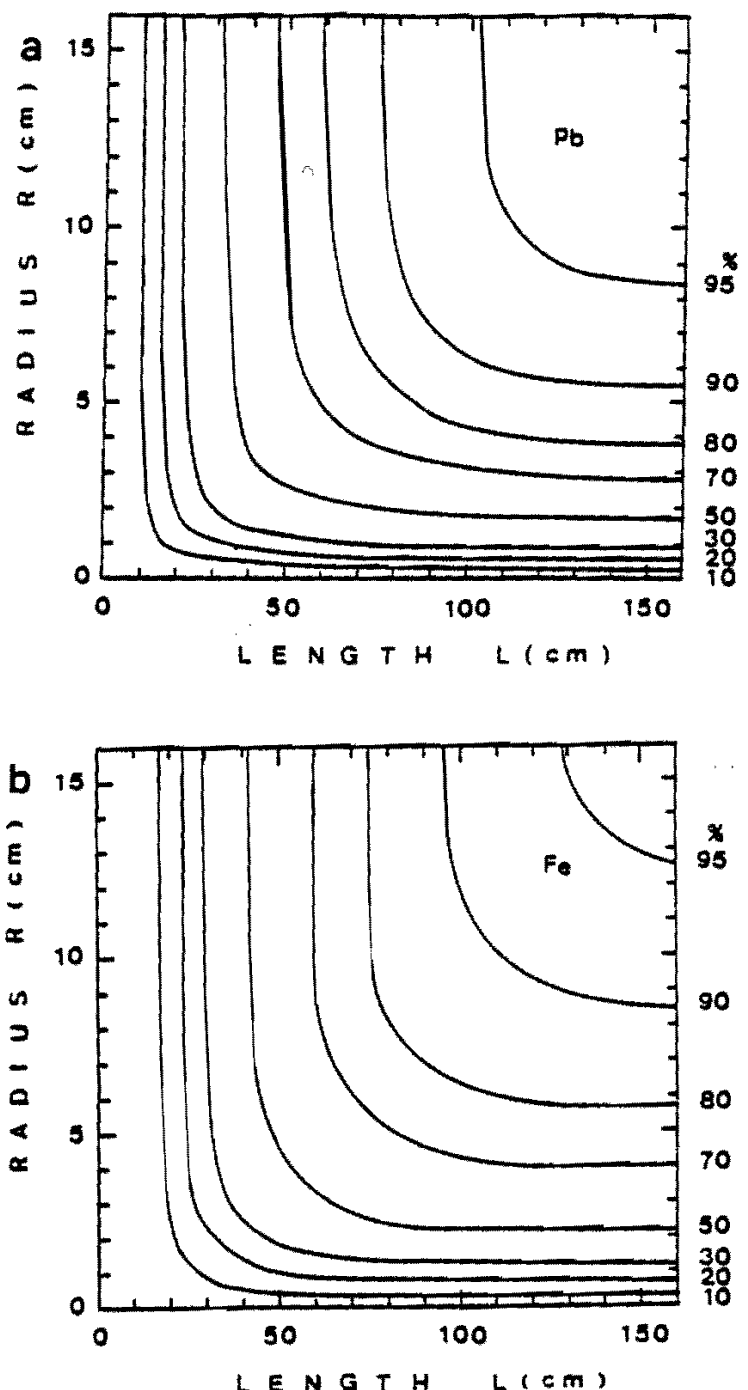

Fig. 9. Energy deposition of shower particles in the cylinder of radius $R$ and length $L$ of (a) lead and (b) iron. Numerals attached to the curves mean the fraction of energy deposition in the cylinder. 
Fig. 5

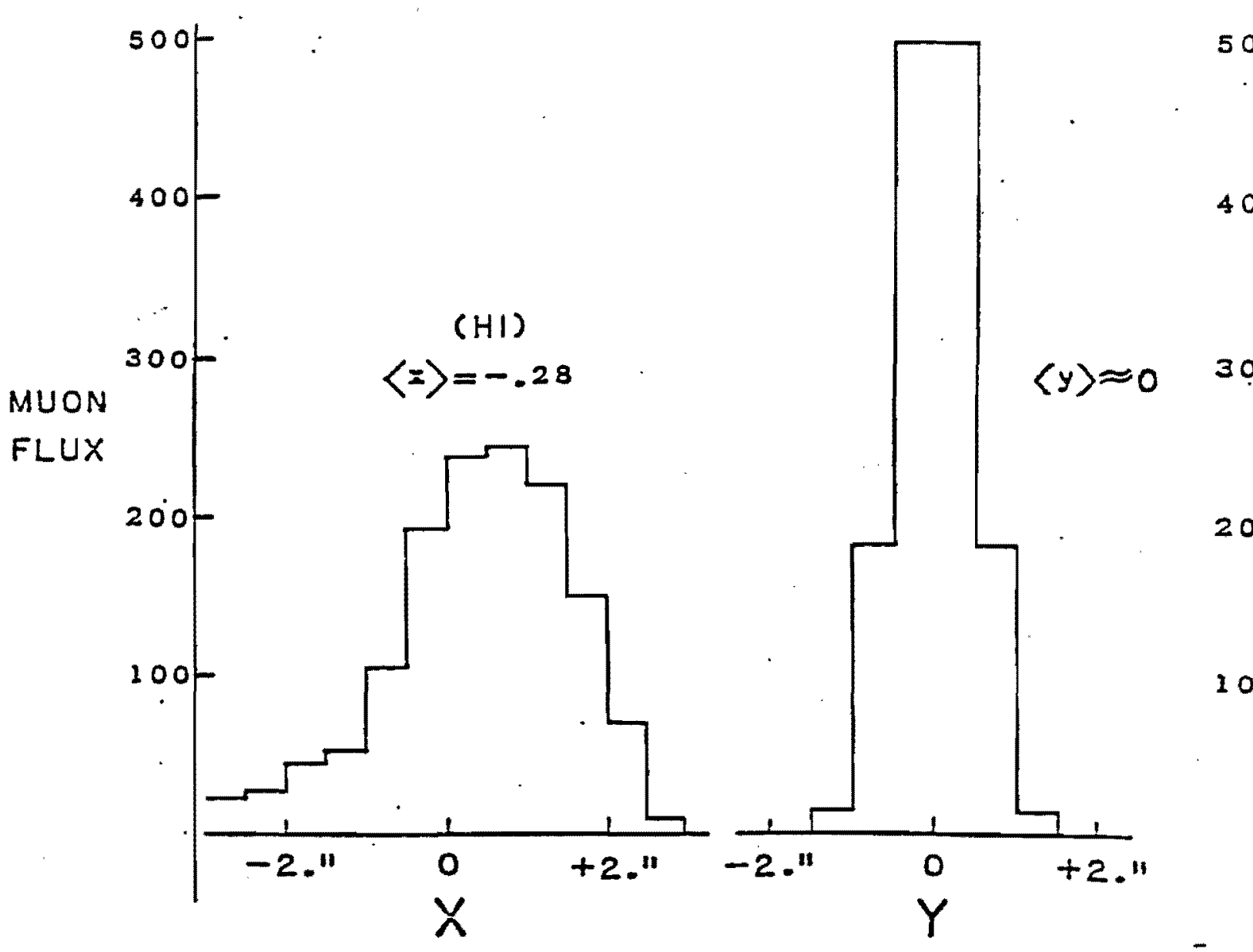




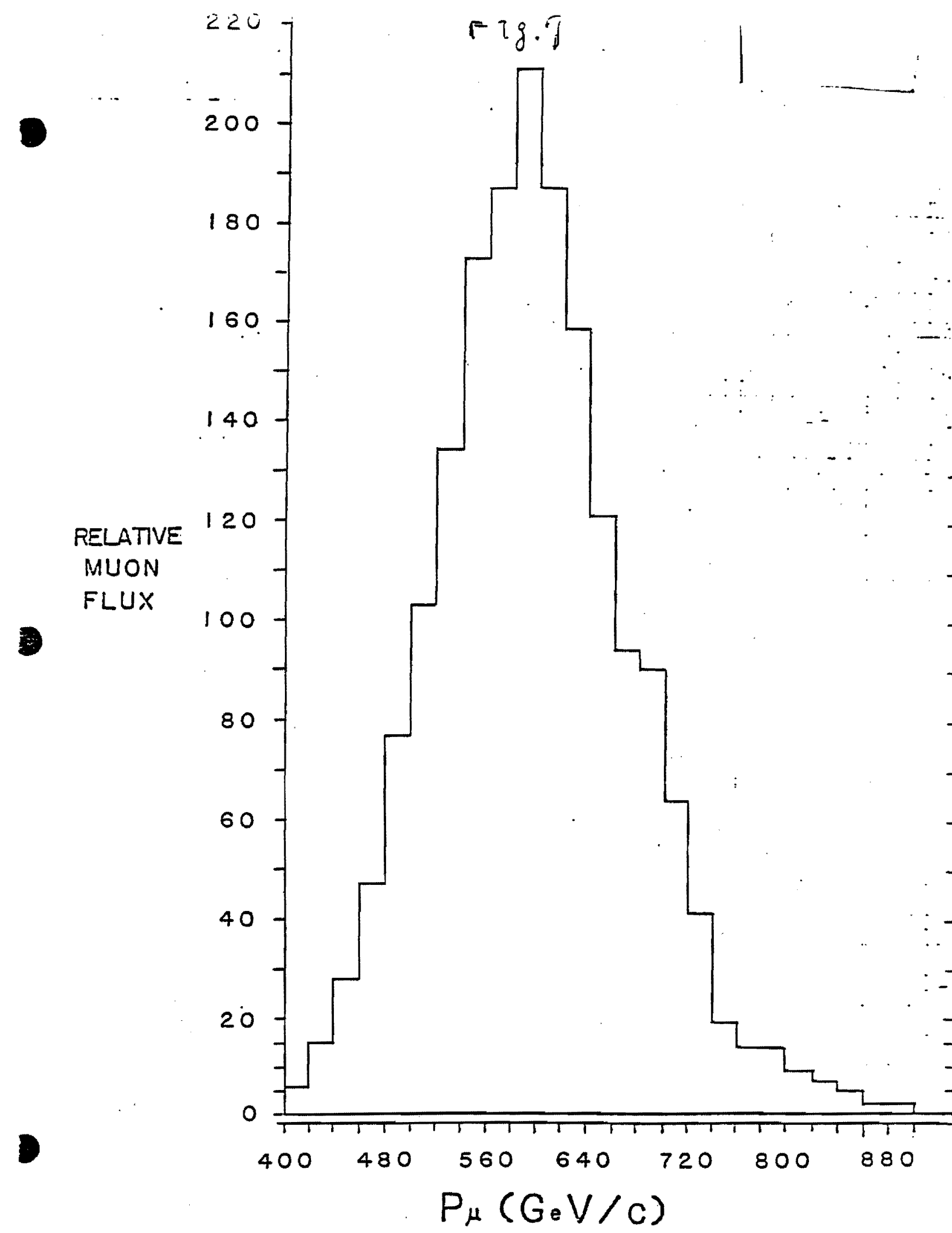

Figure 
Fig. 8

Fe 3om soolayer

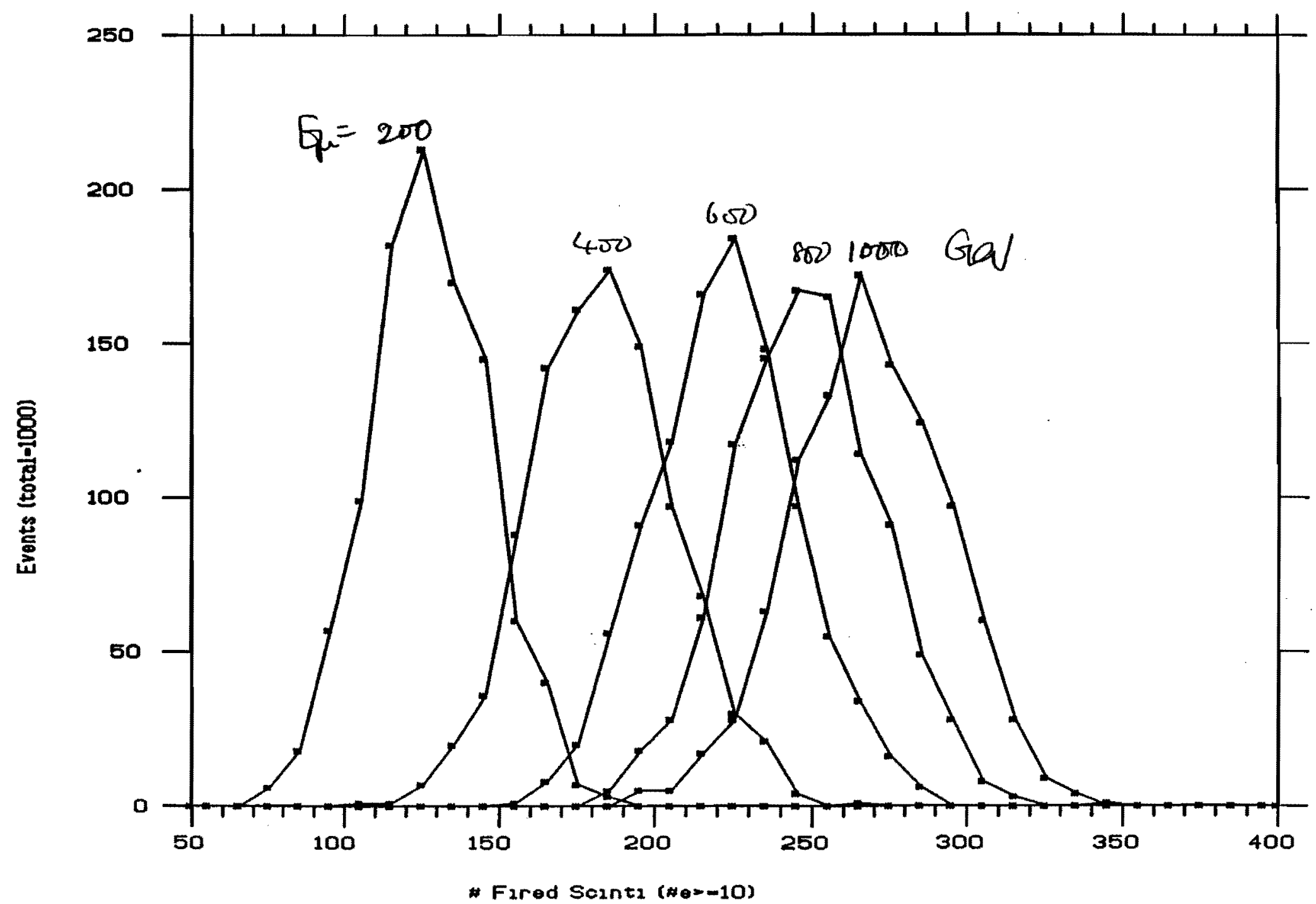


Frg 9

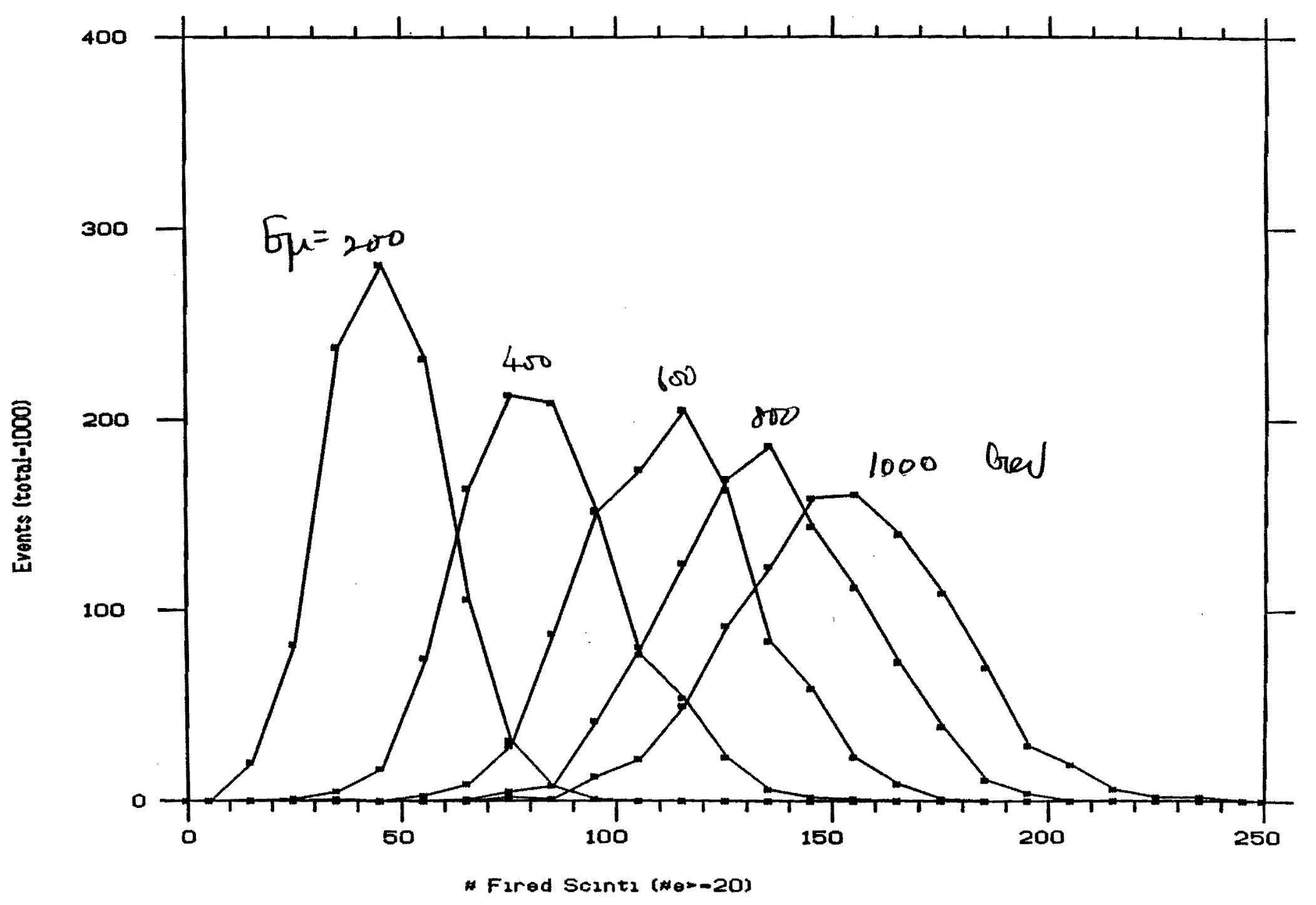

AK 17:42103 22NaV86 